\title{
Research on Application of Virtual Reality Technology in Design and Manufacturing Industry
}

\section{Yanjun Zhang}

Beijing Institute of Science and Technology Information

Beijing, 100044, China

E-mail:s sapple@126.com

\section{Ping Yang ; Liyong Liu ;Anqi Chen}

Beijing Institute of Science and Technology Information

Beijing, 100044, China

E-mail:yangpabjstinfo. com. cn

\begin{abstract}
Virtual reality technology is applied to design and manufacturing industry, which provides new tools and methods, as well as impetus for the entire design and manufacturing industry chain. The technology has formed a large number of typical cases in the fields of automobile, aerospace, home appliances and so forth, greatly manifesting its leading role. With the rapid development and commercialization of virtual reality technology, its application in the design and manufacturing industry will win more popularity, and will highly promote the innovation and development of the design and manufacturing industry at the same time.
\end{abstract}




\section{The Application Characteristics of Virtual Reality in the Design and Manufacturing Industry}

\subsection{The Implementation Model of Repeated Modification}

In the field of design and manufacturing, once the computer virtual model is established, it can interact with the virtual model continuously [1]. The repeated modification of the model is the biggest difference between the virtual and the actual manufacturing processes, and it is this feature that allows virtual manufacturing to quickly change design process and production process [2]. Meanwhile, it assists technicians and managers to design, manage and make decisions, which enables them to correctly keep the balance between the product performance and manufacturing costs, between production schedule and critical decision-making.

\subsection{The Implementation of Distributed Collaborative Cooperation}

The virtual reality technology can achieve distributed collaborative cooperation of the staff and the equipment, break the traditional geographical and time-domain constraints [3]. The technical staff at different locations can cooperate with each other to complete the same virtual manufacturing process through the network, which can promote product development to respond to market changes in a rapid, high-quality and cost effective way. Collaborative design and collaborative assessment at different locations are the main approaches of the current distributed collaborative cooperation.

\subsection{The Implementation of Parallel Working Process}

Parallel work, product design, simultaneous simulation of the processing and assembly process can be realized by virtual design and manufacturing, greatly accelerating the product design process and reducing the trial time for new products. As shown in Figure1, the process of the traditional serial product method is sequential and iterative, which means each step of the product development is carried out in sequence and the product will be handed over from one department to another with long-time development cycle and heavy design workload. Virtual design and manufacturing integrates parallel engineering technology, product design, process design, production technology preparation, procurement and cross production [4]. It makes use of little frequent feedback to replace single feedback by means of virtual reality technology, design-driven manufacturing and manufacturing auxiliary design. Therefore, this method can reflect market demand and manufacturing practice in a more agile way and greatly shorten the product development cycle and reduce development costs [5]. 


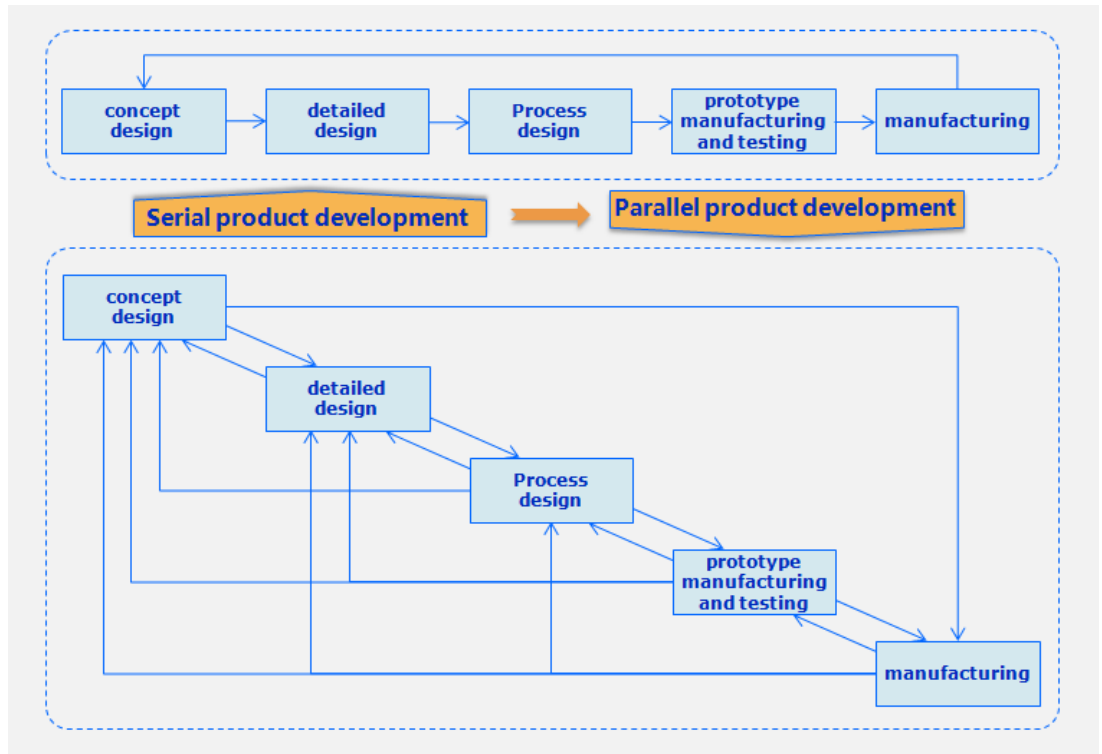

Figure 1 : Comparison of parallel and serial design manufacturing patterns

\section{Application Trend of Virtual Reality in Design and Manufacturing Industry}

\subsection{Industry Applications Becoming More Widely Acknowledged}

The year of 2016 has become the first year of virtual reality industry development, which was driven by the capital with following features: rapid technological progress, continuous emergence of hardware and software products and gradual penetration into the depth of various industries. Virtual reality has been expanded from the application of the national defense and military to various fields such as automobile, aerospace, mechanical equipment, household appliances, furniture and other design and manufacturing areas. China's manufacturing industry scale ranks the first in the world. The number of large-scale manufacturing enterprises is more than 320,000 , which has a wide range of industries, various products, sound industrial systems, and rich application scene of virtual reality technology as well as huge space.

\subsection{The Focus on Design Transferring to Assembly}

The application of virtual reality technology in design and manufacturing industry began with the product appearance design and structural design. With the development of virtual reality technology and the upgrade of the traditional production line assembly process, virtual reality technology is increasingly applied in assembly section that is regarded as the design and manufacturing's back-end [6]. Through the establishment of product digital assembly model, it is possible to create a near virtual environment, replacing the physical prototype of traditional design by virtual products. Product assembly process can be simulated, analyzed, and the assembly performance can be predicted in order to discover potential assembly conflicts and defects then transfer the assembly information to the designer.

\subsection{Expanding Single Application to Full Chain Application}

The application of virtual reality in design and manufacturing industry gradually expands from single application like product design, structural design, and display experience to full chain application such as assembly design, virtual shop layout and product maintenance, which runs through the whole design and manufacturing sectors. Take automobile manufacturing as an 
example, it starts from market research to the installment of one automobile, then introduction to the market, which usually takes two to three years and lots of time in the trial production stage of the car. After applying all-chain virtual reality technology in the automotive design and manufacturing, the cycle from market research to going-to-market of the automobile can be greatly reduced via parallel work [7].

\subsection{The Visualization Systems Will Gradually Go Beyond and Replace Traditional CAD Systems}

The traditional CAD software is the basic tool for design and manufacturing industry, but with the market demand getting higher, user demand becomes more personalized in recent years. Because of the fact that traditional CAD software technology is mainly used in the modeling of product, it fails to meet new demands nowadays. In addition, rising productivity in the life cycle of products about the detailed design process is very limited, so for the market demand for diversification and cost savings requirements, the visualization system will gradually go beyond and replace the traditional CAD systems.

\subsection{Direct Modeling Method Becomes A Hotspot in Technological Research}

Modeling is the key to virtual reality. Various types of sensors provide the humancomputer interaction interface, voice and gesture. Natural user interface is more suitable for interactive modeling of virtual reality in design and manufacturing industry, which is the hotspot of current technological research and application. The research on gesture recognition has been developed to obtain images with depth information through the 3D camera, and depth information has been used to separate the background and objectives to improve the accuracy of target detection and tracking. With the contribution of machine deep learning and the accumulation of big data corpus, voice recognition technology has achieved breakthrough in development and application.

\section{Application of Virtual Reality in Different Designs and Manufacturing Areas}

\subsection{Application of Product Shape Design}

Virtual reality technology can show a product's three-dimensional appearance so that research and development personnel are able to conceive the shape, structure and mold of the product as well as operational program of the parts and further provide the product designers with a convenient way about the product shape. Designers can observe the products from different angles through virtual reality technology. Before the actual production, it can help designers to find the shortcomings of product design for optimization.

Ford has established FiVE immersion virtual reality laboratory in Australia and the United States and several collaborative centers in Germany, India, Brazil and Mexico. By adopting virtual reality, Ford designers check the entire appearance and interior design of the car in a virtual environment, and observe specific details such as console and interior panels. They can also perceive the internal parts of the car, and discuss with the global team about molding to ensure that practicability, consistency and design effects of all the automobile models are repeatedly verified in the simulation environment of the system. 


\subsection{Application of Product Structure Design}

The realization of the product function mainly depends on the sophisticated structural design. In the structural design process, designers should not only conceive a series of related parts to realize the functions, but also consider the compact structure and appearance of the product. They also need to make sure the product safety and reliability with good performance as well as ease at operation with low costs. Virtual reality technology can simulate the structural design to achieve three-dimensional and precise virtual structural design. Especially for complex structure products, design can be done intuitively through virtual reality technology to avoid potential interference and other issues.

European and American enterprises of advanced aviation such as Boeing and Airbus generally adopt virtual reality technology in aircraft design [8]. For example, the Boeing 747 is made of more than 3 million parts, and the overall design of the parts and aircraft is carried out in a virtual reality environment system consisting of hundreds of workstations. Designers wear a display helmet to verify designs in the virtual "aircraft" to exclude structural design errors. Following Boeing 747, Boeing has applied virtual reality technology into the design of 777 and 787 aircrafts. Boeing have completed the design of shape, structure and performance through projection and motion capture techniques of virtual reality, and the deviation between the obtained program and the actual aircraft is less than 1/1000 inches. Statistically, the number of modifications on design errors on Boeing 777 aircraft designed by virtual reality technology is reduced by $90 \%$ the research and development cycle is shortened by $50 \%$, and the cost is reduced by $60 \%$, compared with the traditional design and manufacturing.

\subsection{Application of Product Prototype Performance Evaluation}

For the traditional product design and manufacturing, the product performance cannot be evaluated until the product prototype test, despite the relatively highmanufacturing cost of the prototype. If the product structure is found to be unreasonable after the prototype is done, or some aspects don't meet customer requirements, the prototype must be modified with serious loss. On the other hand, due to the need of minimizing the number of prototypes, designers cannot open their minds, which will largely limit their creativity.

With the help of virtual reality technology, the virtual model can replace the physical prototype to conduct various analysis and testing simulation, and then optimize the structural parameters that affect product functionalities. This will greatly enhance the accuracy of the product performance, reduce the manufacturing and testing costs for a physical prototype, and shorten product development cycle. For instance, Audi automobiles simulate body strength, power system performance, crash performance by the virtual reality technology. The collision test for the Audi Q7 requires one million pieces of data. In the simulated collision experiment, it took 22 hours for the supercomputer to work within 8 processors and the R\&D staff revised the data by the unit of millimeters. The experiment was repeatedly done until the development was completed. The car has experienced about 2,000 virtual collisions before development completion. While using the real car for the test, only 40 tests can be completed during the same time, not to mention the huge cost led by destroying 40 real cars during the collision tests.

\subsection{Application of Product Processing and Manufacturing}

It is hard to find the machinability of products, the selection of machining methods, machining tools and the possible machining defects in machining process during the design, so 
it has to go through simulation and analysis. Through the virtual reality technology, product processing can be simulated, problem can be detected ahead of time, and design modification and other measurements can be done to ensure the target schedule and product quality.

China Yituo Group carried out virtual manufacturing by the digital factory design and simulation software, has made application on big wheel tractor chassis coating lines of the Heilongjiang Industrial Park. They also built up coating line for panel and structural parts, on China National Heavy Industry Luoyang road roller coating line and on disappearing foundry production line. People can test new product casting by virtual reality technology and directly discover casting defects. The finite element analysis and prediction of the deformable parts such as box and thin wall plate are conducted, and the control measures are found to reduce the deformation for improving the manufacturing level.

In addition, the virtual reality technology can also effectively plan the processing materials and combine the related equipment and facilities to obtain safe, efficient and economic operation. The combination of different decision variables, analysis of equipment utilization, system capacity, effective output rate, delivery time, cost and other strategies can stimulate to achieve goals like maximized capacity, optimized schedule and minimized semi-finished goods and inventory.

\subsection{Application of Product Assembly}

Based on the digital solid model of the product, virtual reality technology can improve the quality of product assembly on the aspects of computer analysis, assembly performance verification as well as technological process [9]. Large-scale mechanical products have large numbers of parts, complicated structure and various types. It is difficult to clearly show the assembly of large-scale mechanical products and the relationship between the various parts and components by using of traditional methods. The virtual reality technology is used to establish the model of each part in the computer, which can demonstrate the assembly process of mechanical products. Users can carry out three-dimensional virtual assembly by wearing VR glasses or data gloves.

Yituo Group applies "digital virtual reality display system" which is developed by a local company named Man Heng Digital to create a virtual assembly workshop, which can achieve 360 degree internal panoramic roaming to carry out immersive virtual assembly. Users can not only observe each assembly station from different angles, but also accurately track the production process flow of assembled parts.

\subsection{Application of Product Maintenance and Repair}

Virtual maintenance means that the real maintenance process of the equipment is applied via computer simulation and virtual reality technology to achieve remote real-time virtual detection and maintenance. Virtual reality technology is used to the maintenance of complex systems, which can realize the whole process of inspection from factory to sales. It can break through the space constraints, shorten the time, improve service efficiency, expand service scope, improve service quality and push forward manufacturing services to a new stage.

BMW Motor Company adopts VR smart glasses to guide workers to carry out vehicle maintenance. Through the fault codes stored in the intelligent keys of BMW vehicles, the intelligent glasses can diagnose the faults of the vehicles and generate suggestions for maintenance. While wearing glasses, the workers find the glasses will add colored threedimensional images to indicate construction site, the tools and the maintenance operations that 
need to be done.

Virtual maintenance technology can significantly improve the maintenance status of the equipment, shorten the maintenance time of the product, reduce the production disruption and cost caused by improper decision making in advance, and hence provide a more realistic maintenance environment for quick operation. Virtual maintenance technology can also analyze the equipment failure and maintenance pre-processing, estimate maintenance operations time, configure maintenance resources, select the maintenance tools, make the sequence of equipment parts disassembling and estimate maintenance cost.

\section{Promoting the Virtual Reality in the Design and Manufacturing Industry on the Application of Countermeasures and Suggestions}

At present, the virtual reality technology has been widely used in the global design and manufacturing industry, including China. Virtual reality industry has entered a developing period, and China needs to guide the technology from the aspect of policy, seize the strategic opportunity for the design and manufacturing industry to achieve huge success.

\subsection{Strengthening the Top Design and Improve the Standard Specifications}

The relevant departments should make clear the direction of industrial policy and integration development roadmap between the top design virtual reality and design manufacturing industry. Combing with the design and manufacturing industry development, it is necessary to speed up to improve the virtual reality technology standard system, to strengthen the technical layout and enhance the discourse power of industrial competition.

\subsection{Supporting Technology Research and Development, Product and System Integration}

The related virtual reality sensor performance should be enhanced, therefore to speed up the breakthrough of data processing unit performance like CPU and GPU, and support the rapid data processing requirements of the design and manufacturing industry simulation. At the same time, it is needed to build a complete ecosystem in the application software, content and services, as well as to set up the industrial public service platform integrating technology research, demonstration application, case demonstration, promotion and exchange, and to expand the application scope of virtual reality technology in the design and manufacturing industry.

\subsection{Promoting Industrialization and Industry Applications}

While concentrating on the key parts of virtual reality industry chain, it is required to strengthen cooperation among industry,academia and key technologies, concentrate on resources to make technological breakthrough, and form a sound virtual reality technology system. By making use of policies and financial support, virtual reality technology can be applied on the design and manufacturing areas, combined with China manufacturing 2025 and "Internet +" action plan to support the industrial service innovation as well as model innovation.

\subsection{Optimizing the External Environment for Industrial Development}

It is necessary to strengthen collaborative development for the design and manufacturing industry, study design and manufacturing industry application of virtual reality technology testing and evaluation methods, and establish industrial operation monitoring information 
platform. Full play should be given to industry associations, industry alliances, science research institute in the data statistics, information services, and the relative parties should track the domestic and international technology path and industrial development trends for the development of industry support services to improve market environment for industrial development.

\section{References}

[1] Wang Zhiyu. Application and Design of Intelligent Home $R \&$ D System Based on Virtual Reality [J].Information Communication Press, 2017, (08):13-134.

[2] Shuai Chaolin, Chen Xuemei, Qiu Shiguang.Application and Prospect of Virtual Reality Technology in Aviation Intelligent Manufacturing[J].Aviation Manufacturing Technology Press, 2016, (16):26-33.

[3]Fan Wenjie. Research on Product Design Evaluation based on Immersive Virtual Reality Technology [D].HuaQiao University, 2016.

[4]Yang Qian. Application Status and Development Trend of Virtual Reality Technology in Industrial Design [J].China high tech enterprise press 2015,(16):48-49.

[5] Wang Xiaoyun. Research on Virtual Reality Technology Aided Product Modeling Design [D].Shan Xi University Press, 2014.

[6]Lv Dongxu. The Application and Development of Virtual Realit y on Mechanical Design[J].CaiZhi Press,2013,(19):183.

[7] Zhang Junyou, Jiao Xuejian, Chai Shan.Design and Realization of Vehicle Driving Simulator Based on Virtual Reality [J]. Mechanical Design and Manufacturing Press 2013, (04):39-41.

[8]Zhou Yuhai. Application and Development of Virtual Reality Technology on Advanced Manufacturing Technology [J].Journal of Southern Vocational Education 2012,2(02):23-26.

[9]Zhang Jianmin, Zhang Damin, Chen Jing, Yi Congqin, Ran Hanwei. Virtual Reality Design of Agricultural Machinery_ Based on Digital Media Technology and Ergonomics[J].Agricultural Mechanization Research Press. 2011,33(12):94-97. 